\title{
Chemerin C9 peptide induces receptor internalization through a clathrin-independent pathway
}

\author{
Jun-xian ZHOU ${ }^{1}$, Dan LIAO ${ }^{1}$, Shuo ZHANG ${ }^{1}$, Ni CHENG ${ }^{2}$, Hui-qiong $\mathrm{HE}^{1}$, Richard D YE ${ }^{1,2, *}$ \\ ${ }^{1}$ School of Pharmacy, Shanghai Jiao Tong University, Shanghai 200240, China; ${ }^{2}$ Department of Pharmacology, University of Illinois \\ College of Medicine, Chicago, IL 60612, USA
}

\begin{abstract}
Aim: The chemerin receptor CMKLR1 is one type of G protein-coupled receptors abundant in monocyte-derived dendritic cells and macrophages, which plays a key role in the entry of a subset of immunodeficiency viruses including HIV/SIV into lymphocytes and macrophages. The aim of this work was to investigate how CMKLR1 was internalized and whether its internalization affected cell signaling in vitro.

Methods: Rat basophilic leukemia RBL-2H3 cells, HEK 293 cells, and HeLa cells were used. CMKLR1 internalization was visualized by confocal microscopy imaging or using a FACScan flow cytometer. Six potential phosphorylation sites (Ser337, Ser343, Thr352, Ser344, Ser347, and Ser350) in CMKLR1 were substituted with alanine using site-directed mutagenesis. Heterologous expression of wild type and mutant CMKLR1 allowed for functional characterization of endocytosis, $\mathrm{Ca}^{2+}$ flux and extracellular signal-regulated kinase (ERK) phosphorylation.

Results: Chemerin and the chemerin-derived nonapeptide (C9) induced dose-dependent loss of cell surface CMKLR1-GFP fusion protein and increased its intracellular accumulation in HEK 293 cells and RBL-2H3 cells stably expressing CMKLR1. Up to 90\% of CMKLR1 was internalized after treatment with C9 $(1 \mu \mathrm{mol} / \mathrm{L})$. By using different agents, it was demonstrated that clathrin-independent mechanism was involved in CMKLR1 internalization. Mutations in Ser343 for G protein-coupled receptor kinase phosphorylation and in Ser347 for PKC phosphorylation abrogated CMKLR1 internalization. Loss of CMKLR1 internalization partially enhanced the receptor signaling, as shown by increased $\mathrm{Ca}^{2+}$ flux and a shorter latency to peak level of ERK phosphorylation.

Conclusion: CMKLR1 internalization occurs in a clathrin-independent manner, which negatively regulated the receptor-mediated Ca ${ }^{2+}$ flux and ERK phosphorylation.
\end{abstract}

Keywords: chemerin; CMKLR1; GPCR; receptor trafficking; receptor internalization; endocytosis; calcium flux; ERK phosphorylation

Acta Pharmacologica Sinica (2014) 35: 653-663; doi: 10.1038/aps.2013.198; published online 24 Mar 2014

\section{Introduction}

The human chemerin receptor (ChemR23 or chemokine receptor-like 1, CMKLR1) was first reported as a functionally unknown (orphan) G protein-coupled receptor (GPCR) with close homology to the $\mathrm{C} 3 \mathrm{a}, \mathrm{C} 5 \mathrm{a}$, and formyl peptide receptors, but more distant relation to the subfamilies of chemokine receptors $^{[1,2]}$. CMKLR1 is a coreceptor that plays a key role in the entry of a subset of immunodeficiency viruses, including $\mathrm{HIV} / \mathrm{SIV}$, into lymphocytes and macrophages ${ }^{[2]}$, and its transcripts are abundant in monocyte-derived dendritic cells (DC) and macrophages. The natural ligand for CMKLR1, chemerin, was identified by Wittamer and coworkers ${ }^{[3]}$. Meder and coworkers also identified this ligand and named it as TIG2.

\footnotetext{
* To whom correspondence should be addressed.

E-mail yedequan@sjtu.edu.cn

Received 2013-08-17 Accepted 2013-12-08
}

They reported that this bioactive, circulating TIG2 contains a specific molecular form consisting of amino acid residues 21 to 154 of the 163 amino acid prepropeptide ${ }^{[4]}$. Chemerin is structurally and evolutionarily related to cathelicidin precursors (antibacterial peptides), cystatins (cysteine protease inhibitors), and kininogens ${ }^{[3]}$. Chemerin is secreted as a precursor with low biological activity and is converted to a potent and highly specific agonist for CMKLR $1^{[5]}$ upon proteolytic cleavage of its $\mathrm{COOH}$-terminal domain by a variety of proteases $^{[6]}$. Prochemerin is expressed in many tissues, including the spleen, lymph nodes, and epithelia, and an elevated level of bioactive chemerin was found in a diverse set of human inflammatory fluids. Proteases regulate the bioactivity of this novel mediator during early innate immune responses ${ }^{[7]}$, and primary immune cells, such as human neutrophils, are able to efficiently convert inactive prochemerin into active chemerin ${ }^{[8]}$.

CMKLR1 plays an important role in the recruitment and 
trafficking of leukocytes, including plasmacytoid DC and NK cells ${ }^{[2,9,10]}$. In in vitro studies, chemerin was shown to promote $\mathrm{Ca}^{2+}$ mobilization and chemotaxis of immature DCs and macrophages in a CMKLR1-dependent manner ${ }^{[3]}$. In a more recent study, CMKLR1 was shown to mediate resolvin E1 (RvE1) signaling, although this remains controversia ${ }^{[5]}$. It has been recently reported that binding of RvE1 to CMKLR1 triggers protective and anti-inflammatory actions ${ }^{[11]}$. Furthermore, recombinant chemerin is an adipokine ${ }^{[12]}$ and induces the phosphorylation of extracellular signal-regulated kinases $1 / 2$ (ERK 1/2) and lipolysis in differentiated 3T3-L1 adipocytes ${ }^{[13]}$.

Protease processing of prochemerin generates several peptides ${ }^{[5]}$. The nonapeptide ${ }^{149}$ YFPGQFAFS ${ }^{157}$ (chemerin-9, C9), corresponding to the C-terminus of processed human chemerin minus six amino acids, retains most of the activities of the full-length chemerin ${ }^{[14]}$. Another peptide, termed chemerin-15 (C15; ${ }^{141}$ AGEDPHSFYFPGQFA ${ }^{155}$ ), lacks the bioactivity of full-length chemerin and $C 9^{[15,16]}$, however, it was reported that $\mathrm{C} 15$ displays anti-inflammatory properties ${ }^{[17]}$. The mouse equivalent of $\mathrm{C} 15$ was found to enhance macrophage phagocytosis of microbial particles and apoptotic cells at low picomolar concentrations ${ }^{[18]}$. How $\mathrm{C} 15$ activates CMKLR1 remains to be investigated. Orthologs of chemerin have been unambiguously found in primates, mammals, birds, and fish species. The six cysteines that are presumably involved in disulfide bridge are conserved in all of the species. Additionally, the C-terminal nonapeptide of the mature protein, shown to be important for the biological activity of human chemerin, is highly conserved in all mammalian species examined to date. These findings suggest that the biological activities of chemerin may be conserved across mammalian species $^{[15]}$.

Internalization of GPCRs, which occurs through a cellular process termed endocytosis, plays an important role in receptor trafficking, receptor removal from cell surface, and receptor-mediated signaling ${ }^{[19]}$. Although GPCR internalization may occur constitutively, in most cases it is triggered upon ligand binding. In many published reports, GPCR internalization involves clathrin-coated pits $^{[20]}$, but there is an increasing number of reports showing that GPCRs may also internalize through clathrin-independent mechanisms, such as those involving caveolae ${ }^{[21]}$. Early studies have shown that phosphorylation of GPCR in its intracellular loop may be mediated by $G$ protein-coupled receptor kinase (GRK), protein kinase $\mathrm{C}$ (PKC) or protein kinase A (PKA) and is critical for its binding to $\beta$-arrestins for the termination of GPCR signaling (desensitization) and receptor internalization ${ }^{[22-24]}$. Internalization of CMKLR1 has been reported ${ }^{[25,26]}$, but has not been carefully characterized. We sought to determine what mechanisms were used for the peptide agonist-induced CMKLR1 internalization. Our results showed that CMKLR1 internalization is triggered by chemerin and the $\mathrm{C} 9$ peptide, but not by the $\mathrm{C} 15$ peptide. A clathrin-independent pathway is involved in CMKLR1 internalization. Moreover, CMKLR1 internalization is not required for ERK phosphorylation, and abolishing CMKLR1 internalization alters the kinetics of C9-induced ERK phosphorylation.

\section{Materials and methods Reagents}

Cell culture media were obtained from Hyclone (Beijing, China). Fetal bovine serum and Lipofectamine 2000 reagent were purchased from Life Technologies (Carlsbad, CA, USA). Recombinant human chemerin was purchased from R\&D Systems (Minneapolis, MN, USA). The C9 derived from human chemerin was synthesized by ShineGene Molecular Biotech, Inc (Shanghai, China); the C15 and a scrambled C15 peptide $\left(\mathrm{sC} 15,{ }^{141}\right.$ DPSQAPFEGFHAFGY ${ }^{155}$ ) were synthesized by ChinaPeptides Co, Ltd (Shanghai, China). Anti-phospho-ERK1/2 and anti-ERK1/2 antibodies were purchased from Cell Signaling Technology (Danvers, MA, USA). FLIPR Calcium 5 reagent was purchased from Molecular Devices (Sunnyvale, CA, USA).

\section{Cell culture, plasmid preparation and transfection}

HeLa cells (CRM-CCL2, ATCC, Manassas, VA, USA) were maintained in Dulbecco's modified Eagle's medium supplemented with $10 \%$ heat-inactivated fetal bovine serum. Fulllength human CMKLR1 cDNA was subcloned into the expression plasmids pcDNA3.1(+) for transfection into HEK 293 and SFFV.neo for transfection into RBL-2H3. A CMKLR1-GFP fusion construct was also prepared, by in-frame fusion of fulllength CMKLR1 coding sequence to that of enhanced green fluorescent protein (EGFP; minus the first codon for methionine), and subcloned into the EcoRI site of the pcDNA3.1(+) vector. Site-directed mutagenesis was performed on the cDNA of human CMKLR1 and the mutations were verified by DNA sequencing. HeLa cells were transfected with the expression plasmids using Lipofectamine 2000 according to manufacturer's instructions. The cells were incubated at $37^{\circ} \mathrm{C}$ for 24-36 $\mathrm{h}$ after transfection prior to performing the functional assays.

To generate stable transfectants, the rat basophilic leukemia RBL-2H3 cell line (CRL-2256, ATCC) and the human kidney epithelial HEK 293 cell line (CRL-1573, ATCC) were transfected with the CMKLR1-SFFV.neo and CMKLR1pcDNA3.1(+) plasmids, respectively, using Lipofectamine 2000. Stable transfectants were selected with $500 \mu \mathrm{g} / \mathrm{mL}$ G418 (Life Technologies) and were maintained in the presence of $250 \mu \mathrm{g} / \mathrm{mL}$ G418.

\section{Receptor internalization and fluorescence microscopy}

HeLa cells were grown on polylysine-treated glass coverslips to $60 \%-80 \%$ confluency. The cells were transiently transfected with the CMKLR1-EGFP expression plasmids (wild type and mutants). After $24 \mathrm{~h}$, the medium was removed, and the cells were treated with the ligands (C9, C15 or recombinant chemerin) in PBS with $0.1 \%$ BSA. Internalization was carried out at $37^{\circ} \mathrm{C}$ for the the indicated time periods. The ligand was then removed, and the cells were fixed with $4 \%$ paraformaldehyde for $20 \mathrm{~min}$. After washing three times with PBS, the cells were incubated with $5 \mu \mathrm{g} / \mu \mathrm{L}$ DAPI for $5 \mathrm{~min}$ in the dark at 
room temperature. To determine the role of clathrin in receptor internalization, the dynamin I mutant S45N was transiently transfected into HEK 293 cells together with the CMKLR1EGFP fusion construct. When indicated, the cells were pretreated for $30 \mathrm{~min}$ with pharmacological inhibitors including $100 \mathrm{mmol} / \mathrm{L}$ monodansylcadaverine (MDC, Sigma-Aldrich, St Louis, MO, USA), $0.5 \mathrm{~mol} / \mathrm{L}$ sucrose (Sinopharm, Shanghai, China), or $5 \mu \mathrm{g} / \mathrm{mL}$ Filipin III (Sigma-Aldrich), prior to agonist stimulation.

\section{Flow cytometry}

RBL-2H3 cells stably expressing human CMKLR1 were harvested, washed with ice-cold PBS, and resuspended at a density of $1 \times 10^{6}$ cells $/ \mathrm{mL}$ on ice in extracellular buffer (HBSS plus $0.5 \% \mathrm{BSA}$ and $20 \mathrm{mmol} / \mathrm{L}$ HEPES, $\mathrm{pH}$ 7.4). The cells were treated with $\mathrm{C} 9$ at concentrations ranging from $0.1 \mathrm{nmol} / \mathrm{L}$ to $1 \mu \mathrm{mol} / \mathrm{L}$ for $25 \mathrm{~min}$ at $37^{\circ} \mathrm{C}$. After fixation, the cells were incubated for $1 \mathrm{~h}$ with mouse anti-human CMKLR1 antibody (1:200, R\&D Systems), or normal mouse IgG as a negative control (1:200, Santa Cruz Biotechnology, Dallas, TX, USA), followed by incubation with a FITC-labeled goat anti-mouse IgG $(\mathrm{H}+\mathrm{L})$ secondary antibody (1:500; Boster, Wuhan, China) for $1 \mathrm{~h}$ on ice. The residual receptors on the cell surface were detected using a FACScan flow cytometer (Becton Dickinson, Franklin Lakes, NJ, USA).

\section{Calcium mobilization}

HEK 293 cells were transiently transfected with expression constructs encoding human CMKLR1 or the individual point mutants. Stable transfectants of RBL-2H3 cells expressing each of the mutants were also generated. The cells were grown to $90 \%$ confluency in black wall/clear bottom 96-well assay plates. The transiently transfected cells were treated with FLIPR Calcium 5 reagent (Molecular Devices) for $1 \mathrm{~h}$ at $37^{\circ} \mathrm{C}$. The addition of $1 \mathrm{nmol} / \mathrm{L}$ C9 ligand was robotically controlled and the samples were read using a FlexStation III multireader (Molecular Devices). The cells were excited at 485 $\mathrm{nm}$ and were detected at an emission wavelength of $525 \mathrm{~nm}$. Data were acquired using SoftMax Pro 6 software (Molecular Devices).

\section{MAP kinase assays and western blotting analysis}

Transiently transfected HeLa cells expressing either the wild type (WT) or mutant forms of CMKLR1 were harvested, resuspended at a density of $8 \times 10^{6}$ cells/mL in PBS, and stimulated with $10 \mathrm{nmol} / \mathrm{L} \mathrm{C} 9$ at $37^{\circ} \mathrm{C}$ for the indicated time. The samples were lysed in Laemmli sample buffer. Aliquots of the whole cell extracts were separated on 12\% SDS-PAGE and then electroblotted onto PVDF membranes (EMD Millipore Corporation, Billerica, MA, USA). ERK and phosphorylated ERK (p-ERK) were detected by anti-ERK antibodies and anti-phospho-ERK antibodies (Cell Signaling Technologies), respectively.

\section{Statistical analysis}

The data were analysed using Prism software (ver 5.0,
GraphPad, San Diego, CA, USA) and are displayed as the mean $\pm S E M$ of at least 3 independent experiments. Statistical significance was calculated using Student $t$-test, and a $P$ value less than 0.05 was considered significant.

\section{Results \\ Cell surface expression and agonist-induced internalization of CMKLR1}

The majority of GPCRs are cell surface receptors that undergo internalization upon agonist stimulation ${ }^{[20]}$. To determine the internalization properties of CMKLR1, a full-length CMKLR1 cDNA was fused to a cDNA of enhanced green fluorescent protein (EGFP), generating a CMKLR1-EGFP fusion construct. Stable HEK 293 transfectants expressing CMKLR1EGFP were prepared with G418 selection. As shown in Figure 1A, CMKLR1-EGFP was detected at the cell periphery in the absence of an agonist, indicating the cell surface expression of the fusion protein in the resting cells. Stimulation of the transfectants with as little as $1 \mathrm{nmol} / \mathrm{L}$ of the chemerinderived nonapeptide C9 led to the internalization of the receptors, which was shown as intracellularly trapped green fluorescence. Efficient and consistent internalization of the receptor was observed at $100 \mathrm{nmol} / \mathrm{L}$. Receptor internalization appeared as early as $15 \mathrm{~min}$ after C9 stimulation (data not shown). As a result, the internalization assays were carried out for 20-25 min to ensure sufficient uptake of the ligandbound receptors.

To exclude the possible interference of the EGFP molecule on receptor internalization, full-length CMKLR1 was expressed without the EGFP fusion in stably transfected rat basophilic leukemia (RBL) cells. The cell surface expression of CMKLR1 in the stable cell line was detected using an antibody against the receptor, followed by a FITC-labeled secondary antibody. Flow cytometry fluorescence detection confirmed the cell surface expression of CMKLR1 (Figure 2A). Internalization of CMKLR1 was shown as a loss of cell surface fluorescence after incubation with the agonist prior to antibody detection. The C9 peptide dose-dependently induced CMKLR1 internalization at concentrations ranging from $1 \mathrm{nmol} / \mathrm{L}$ to $1 \mu \mathrm{mol} / \mathrm{L}$ (Figure 2A). At the highest concentration used, approximately $90 \%$ of the cell surface CMKLR1 was internalized based on the loss of detectable fluorescence.

\section{CMKLR1 is internalized via a clathrin-independent pathway}

Endocytosis of a large number of GPCRs is mediated through clathrin-coated pits ${ }^{[20]}$. We sought to determine whether CMKLR1 internalization requires clathrin. The stable HEK293CMKLR1-EGFP cell line was treated with monodansylcadaverine (MDC), an inhibitor of the transglutaminase involved in the internalization of various receptors ${ }^{[27]}$, or with sucrose for $30 \mathrm{~min}$ to prevent the formation of clathrin-coated pits. As shown in Figure 1B, these agents did not prevent the internalization of CMKLR1-EGFP. Likewise, expression of dynamin $S 45 \mathrm{~N}$, a dominant negative mutant of dynamin I, which is required for the formation of endocytic vesicles ${ }^{[28]}$, did not interfere with CMKLR1-EGFP internalization (Figure 1B). To 
A
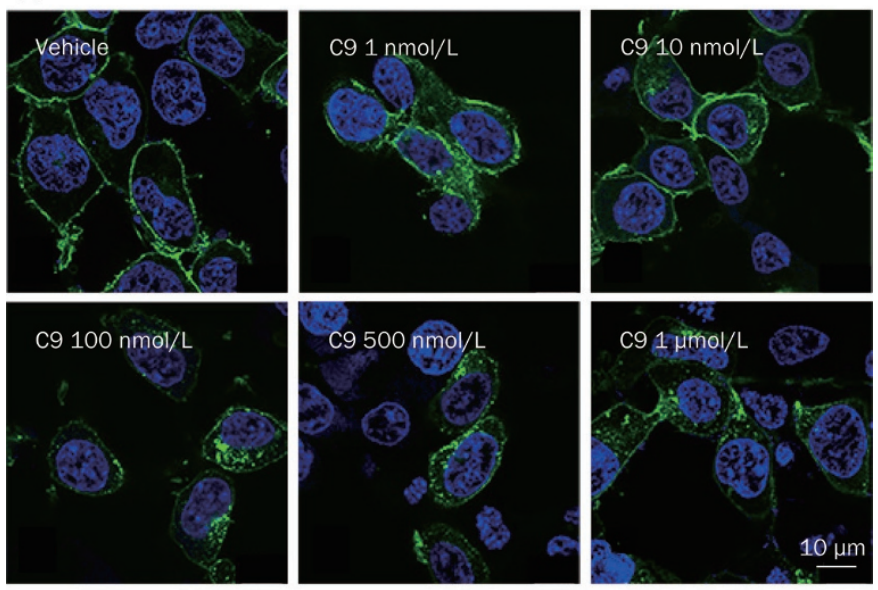

B
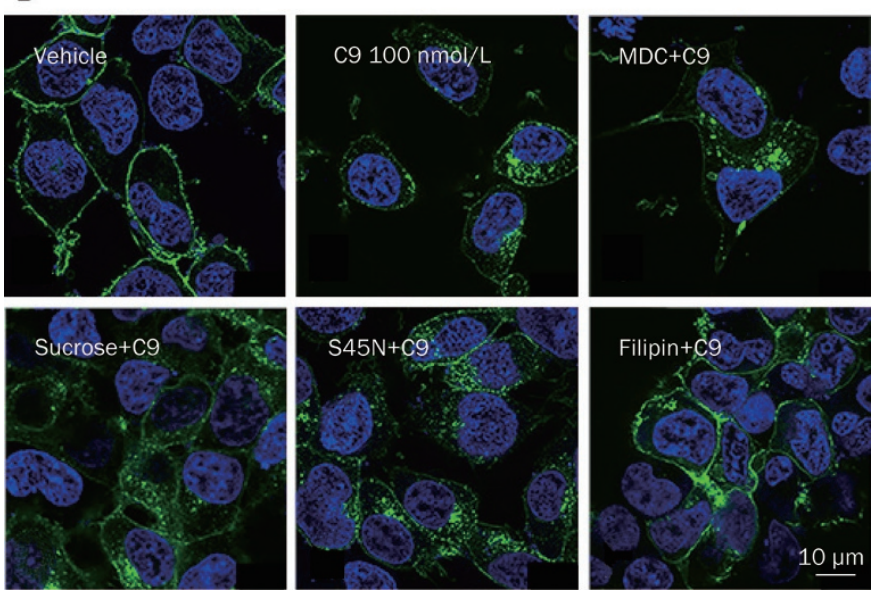

Figure 1. Agonist-induced internalization of CMKLR1 in transfected HEK 293 cells. (A) CMKLR1-EGFP internalization in stably transfected HEK 293 cells was visualized by confocal microscopy imaging 25 min after treatment with different concentrations of the chemerin-derived nonapeptide (C9). The images shown are representative of at least 3 independent experiments with similar results. (B) The HEK293-CMKLR1-EGFP cells were pretreated with MDC $(100 \mathrm{mmol} / \mathrm{L})$ or sucrose $(0.5 \mathrm{~mol} / \mathrm{L})$ for $30 \mathrm{~min}$, or co-transfected with the dynamin I mutant $\mathrm{S} 45 \mathrm{~N}$, to inhibit internalization through clathrin-coated pits. For comparison, HEK293-CMKLR1-EGFP cells were also pretreated with Filipin III $(5 \mu \mathrm{g} / \mathrm{mL})$ to disrupt caveloae. At this concentration, Filipin III did not significantly alter cell viability or migration (data not shown). After treatment, the cells were stimulated with $\mathrm{C9}$ (100 nmol/L) for $25 \mathrm{~min}$. For cells expressing the S45N mutant, C9 was added $36 \mathrm{~h}$ after transient transfection. CMKLR1-EGFP internalization was assessed by confocal microscopy imaging as above. Scale bar=10 $\mu \mathrm{m}$.

verify that the EGFP fusion did not alter CMKLR1 internalization, CMKLR1-RBL cells were treated with sucrose or MDC, and the loss of cell surface fluorescence was measured using flow cytometry as described above. Neither sucrose nor MDC altered the pattern of receptor internalization (Figure 2B). These results suggest that internalization of CMKLR1 might not be mediated through clathrin-coated pits. We next investigated whether caveolae were involved in the endocytosis
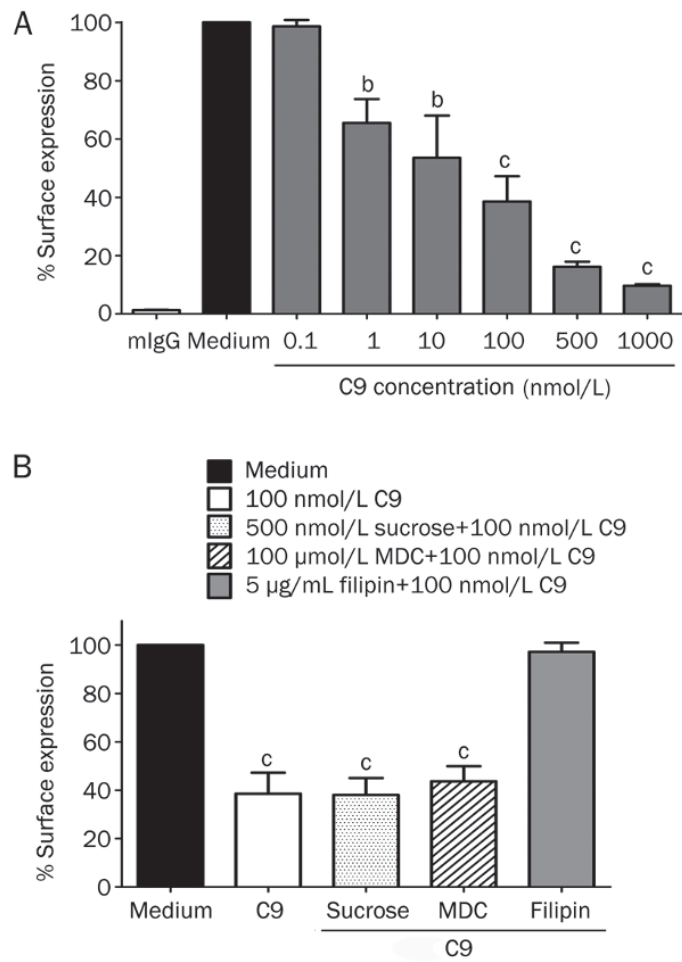

Figure 2. Effects of clathrin-coated pit and caveolae on CMKLR1 internalization in transfected RBL cells. (A) RBL-2H3 cells stably expressing CMKLR1 (CMKLR1-RBL) were treated with vehicle (medium) or C9 peptide for $25 \mathrm{~min}$ at the indicated concentrations. An anti-human CMKLR1 antibody and FITC-conjugated secondary antibody were added to detect CMKLR1 that remained on cell surface by flow cytometry. The loss of cell surface CMKLR1 was calculated based on the geometric mean intensity of fluorescence; the vehicle (medium)-treated sample was set as 100, and the normal mouse IgG (mlgG) control was set as 0 . The data represent the mean \pm SEM from 3 independent experiments. ${ }^{b} P<0.05$ and ${ }^{\mathrm{C}} P<0.01$ compared to the control (medium). (B) CMKLR1-RBL cells were pretreated with the indicated inhibitors or vehicle (medium) as described in Figure 1B. The cells were then stimulated with $100 \mathrm{nmol} / \mathrm{L}$ of $\mathrm{C} 9$ peptide to induce receptor internalization. The loss of cell surface CMKLR1 was calculated as above. The data represent the mean \pm SEM from 3 independent experiments. ${ }^{\mathrm{c}} P<0.01$ vs medium only.

of CMKLR1. The cells were treated with Filipin III, a known inhibitor of the caveola-mediated endocytosis pathway ${ }^{[29,30]}$. Treatment with $5 \mu \mathrm{g} / \mathrm{mL}$ Filipin III blocked the internalization of CMKLR1-EGFP (Figure 1B), resulting in a complete reversal of the loss of cell surface CMKLR1 in the CMKLR1-RBL cells (Figure 2B). These results suggest that CMKLR1 belongs to a group of GPCRs that are compartmentalized through caveo$\operatorname{lae}^{[21]}$.

Effects of chemerin and chemerin-derived peptides on receptor internalization

HeLa cells express the recombinant CMKLR1 at lower levels than HEK 293, allowing better separation of cell surface receptors from those inside the cells. We first determined whether CMKLR1 could be internalized in HeLa cells. Chemerin and 
the C9 peptide induced receptor internalization at $10 \mathrm{nmol} / \mathrm{L}$ (20 min) (Figure 3). The C15 peptide, which is derived from a stretch of human chemerin sequence $\left({ }^{141}\right.$ AGEDPHSFYFPGQFA $^{155}$ ) and does not possess agonistic activity ${ }^{[15,16]}$, failed to stimulate CMKLR1 internalization at $10 \mathrm{nmol} / \mathrm{L}$. When the cells were first incubated with the C15 peptide for $10 \mathrm{~min}$ and then stimulated with the C9 peptide for $20 \mathrm{~min}$, internalization of CMKLR1 was blocked. The same effect was seen with C15 pretreatment followed by chemerin stimulation. A scrambled C15 (sC15, ${ }^{141}$ DPSQAPFEGFHAFGY ${ }^{155}$ ) did not stimulate receptor internalization and did not prevent C9- or chemerininduced CMKLR1 internalization. These results suggest that the C15 peptide antagonize CMKLR1 internalization, but it is presently unclear whether this blocking effect occurs at the receptor or ligand level.

Identification of the structural determinants for agonist-induced internalization of CMKLR1

The intracellular domains of GPCRs contain Ser and Thr that are phosphorylated ${ }^{[22,23]}$ and serve as binding sites for $\beta$-arrestins ${ }^{[24]}$. The GRKs are the major kinases that phosphorylate the GPCRs ${ }^{[31,32]}$. Additionally, GPCRs may be phosphorylated by other kinases including PKA and PKC ${ }^{[22,33]}$. Computer analysis ${ }^{[34]}$ of the CMKLR1 sequence identified six potential sites for GRK phosphorylation within the predicted cytoplasmic tail (Figure 4 and Table 1). Potential phosphorylation sites for PKB (Akt) and PKC were also identified (Table $1)$. We selected two of the five predicted GRK phosphorylation sites (Ser337 and Ser343), one of the predicted PKB phos-
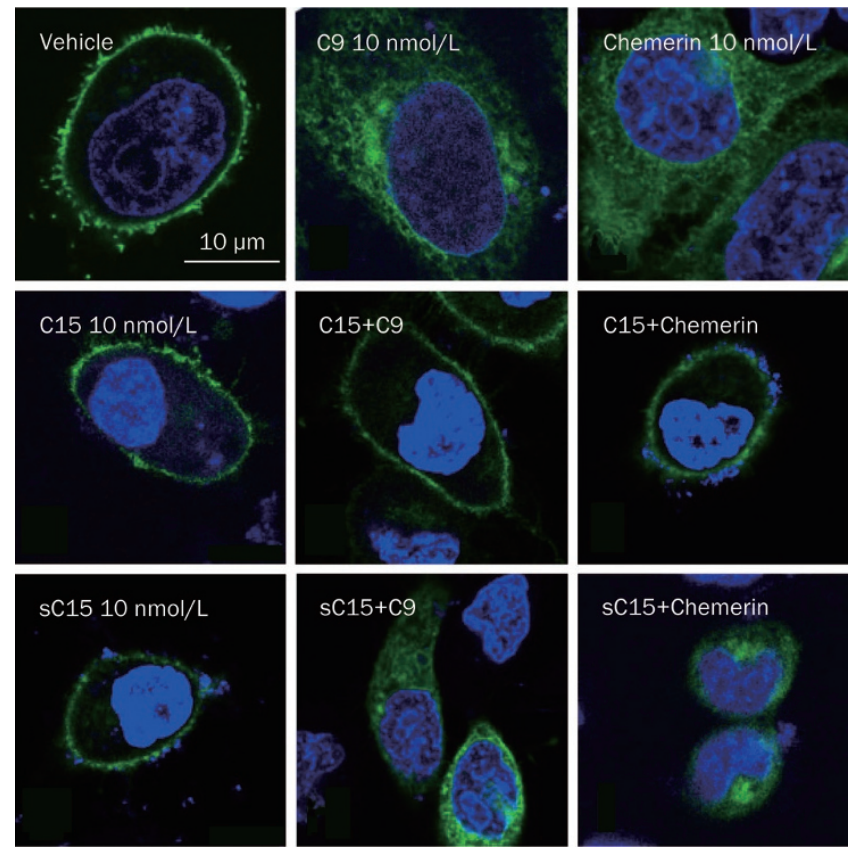

Figure 3. Effects of chemerin and chemerin-derived peptides on CMKLR1 internalization. CMKLR1-EGFP internalization was determined in transiently transfected HeLa cells after stimulation with vehicle, C9, chemerin, C15, C15 then C9, C15 then chemerin, sC15, sC15 then $\mathrm{C} 9$, and $\mathrm{sC} 15$ then chemerin. All ligands were used at $10 \mathrm{nmol} / \mathrm{L}$ and each stimulation lasted for $20 \mathrm{~min}$. Receptor internalization was determined by confocal microscopy imaging. Representative images from at least 3 independent experiments are shown. Scale bar=10 $\mu \mathrm{m}$.

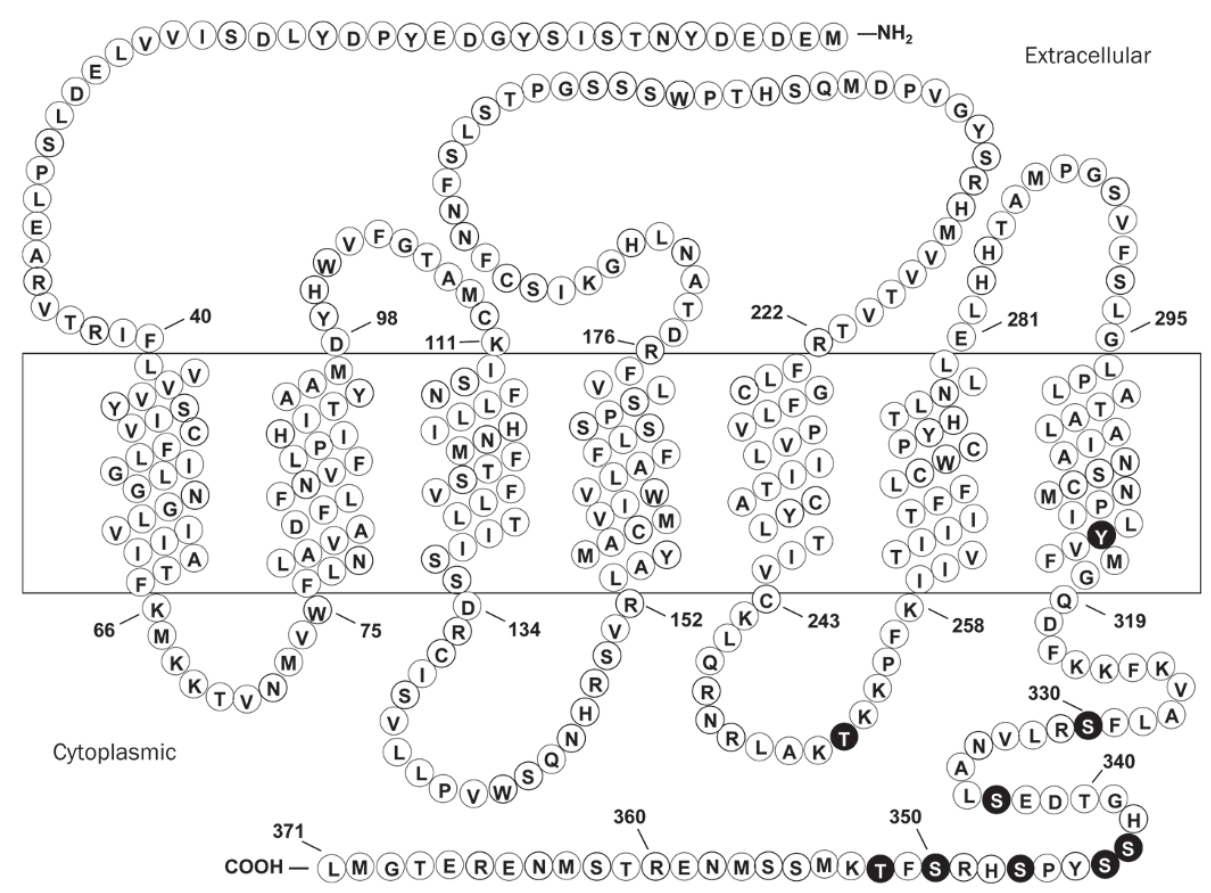

Figure 4. Schematic representation of the CMKLR1 sequence and sites of mutagenesis. A schematic drawing of the seven transmembrane domain structure of CMKLR1 is shown. The point mutation sites are marked with the reverse color. 
Table 1. Potential sites for phosphorylation in CMKLR1.

\begin{tabular}{|c|c|c|c|c|c|c|}
\hline Position & Kinase & Sequence & $\begin{array}{l}\text { Thre- } \\
\text { shold }\end{array}$ & $\begin{array}{l}\text { Risk- } \\
\text { Diff }\end{array}$ & Mutation & $\begin{array}{l}\text { Endo- } \\
\text { cytosis }\end{array}$ \\
\hline 253 & & RLAKTKKPF & & & T253A & - \\
\hline 314 & & NPILYVEMG & & & Y314A & - \\
\hline 330 & & VALFSRLVN & & & S330A & + \\
\hline 337 & GRK & VNALSEDTG & 2.1 & 3.04 & S337A & + \\
\hline 343 & GRK & DTGHSSYPS & 2.1 & 3.13 & S343A & - \\
\hline 344 & PKC & TGHSSEYPSH & 2.2 & 2.37 & S344A & + \\
\hline 347 & PKC & SSYPSHRSE & 2.2 & 3.37 & S347A & - \\
\hline 350 & PKC & PSHRSETKM & 2.2 & 3.15 & S350A & + \\
\hline 352 & PKB & HRSFTKMSS & 3.2 & 4.86 & T352A & + \\
\hline
\end{tabular}

The PPSP program ${ }^{[34]}$ was used for an analysis of the protein sequence of human CMKLR1. Potential sites for phosphorylation by GRK, PKB (Akt), and PKC were underlined. Selected sites located in the intracellular domains, particularly in the cytoplasmic tail, are listed in the table. The last two columns show the mutations introduced and their effects on receptor internalization. phorylation sites (Thr352) and three of the PKC phosphorylation sites (Ser344, Ser347, and Ser350) for alanine substitution using site-directed mutagenesis. Additionally, two serines and threonines that did not match any consensus phosphorylation sequence were chosen for comparison (Ser330 in the C-terminal domain and Thr253 in the third intracellular loop). All of the mutants were expressed on the surface of the transiently transfected HeLa cells (Figure 5), indicating that none of the point mutations caused significant changes in the global structure of CMKLR1. Moreover, the mutant receptors were able to mediate $\mathrm{Ca}^{2+}$ flux (Figure 6), indicating that their entire structures were retained.

In addition to the above-mentioned potential phosphorylation sites, point mutations were generated within the NPXXY motif, which is known to be important for GPCR internalization $^{[35]}$. One of the mutants, Y314A, was analyzed together with the phosphorylation site mutants using confocal microscopy. As expected, alanine substitution abrogated the internalization of the Y314A mutant receptor (Figure 5). Additionally, alanine substitution at positions 343 (predicted GRK site)
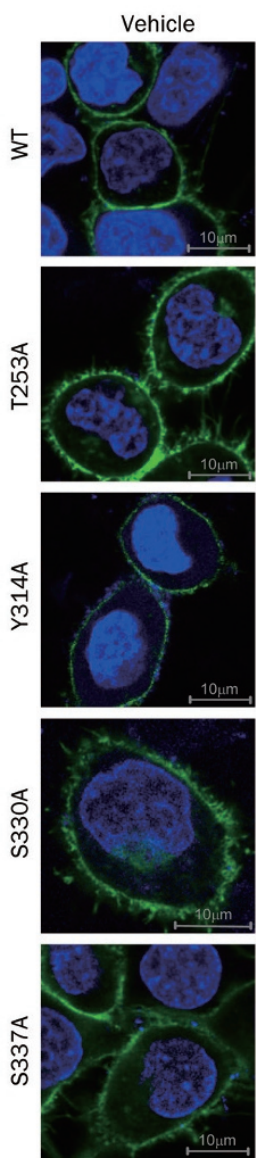

C9
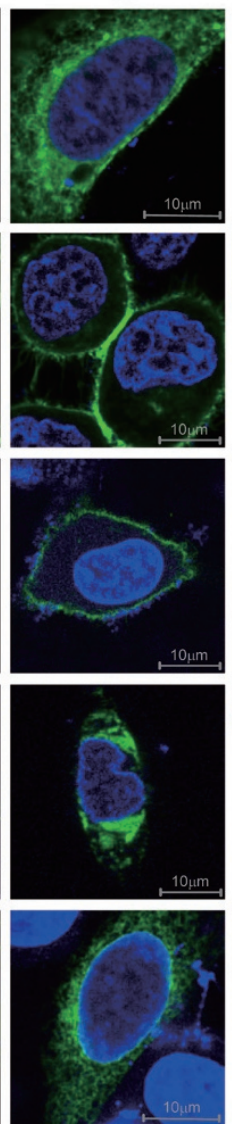

Chemerin
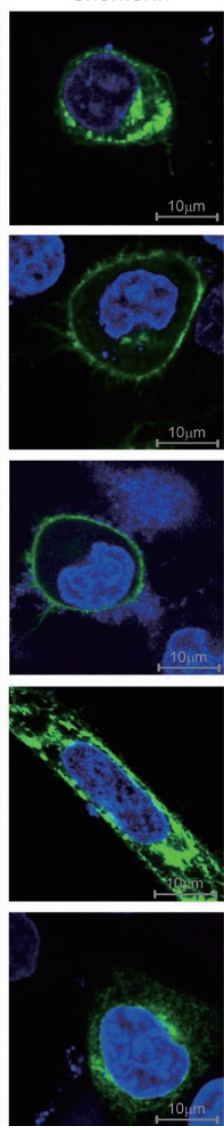
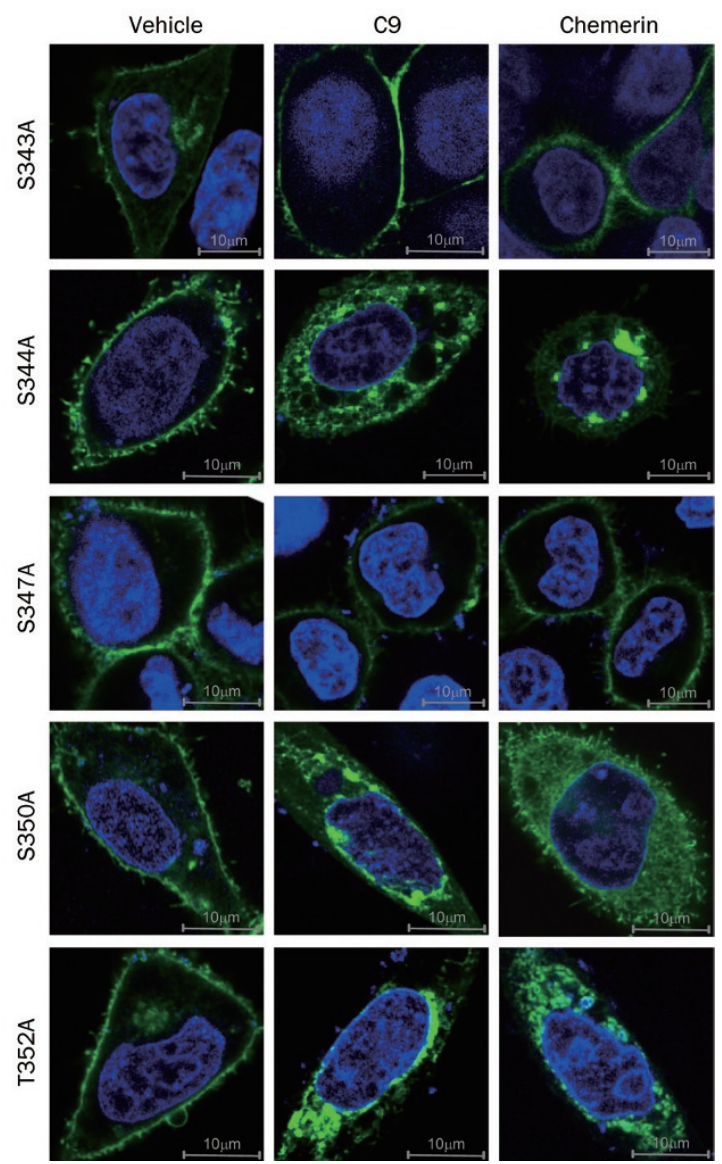

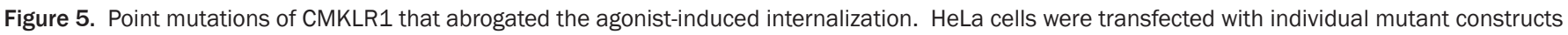

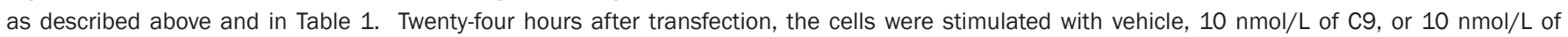

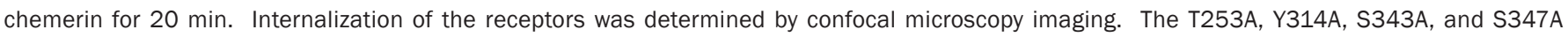

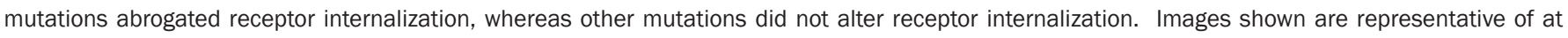
least three independent experiments. Scale bar=10 $\mu \mathrm{m}$. 
A
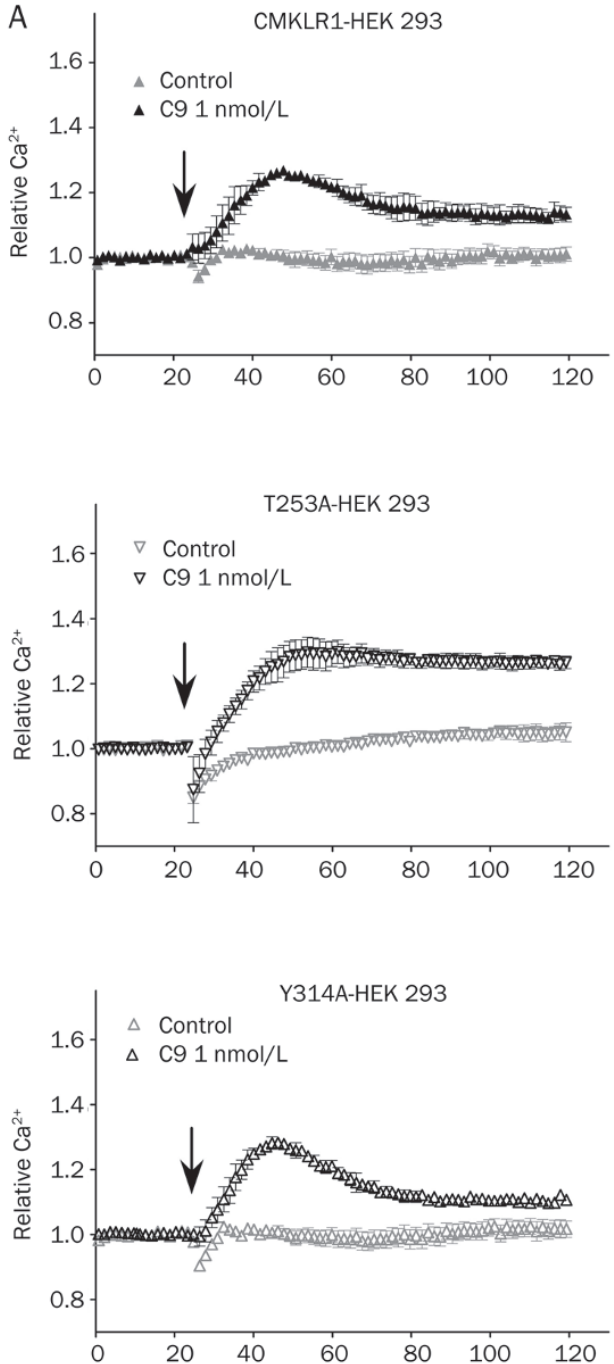

S347A-HEK 293

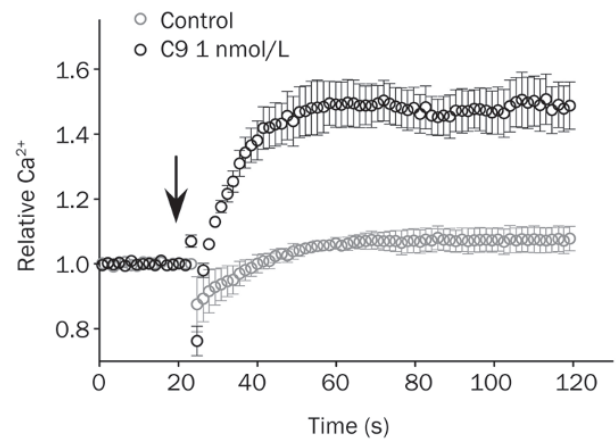

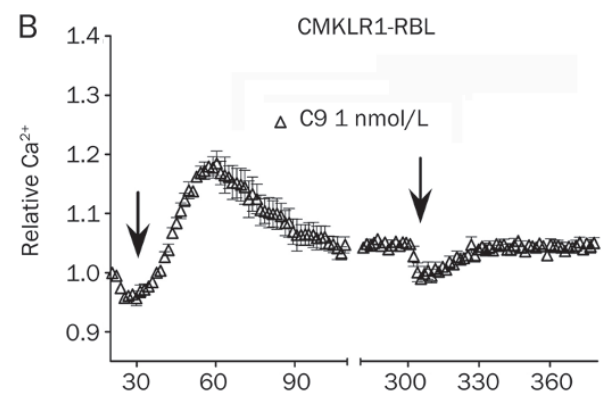
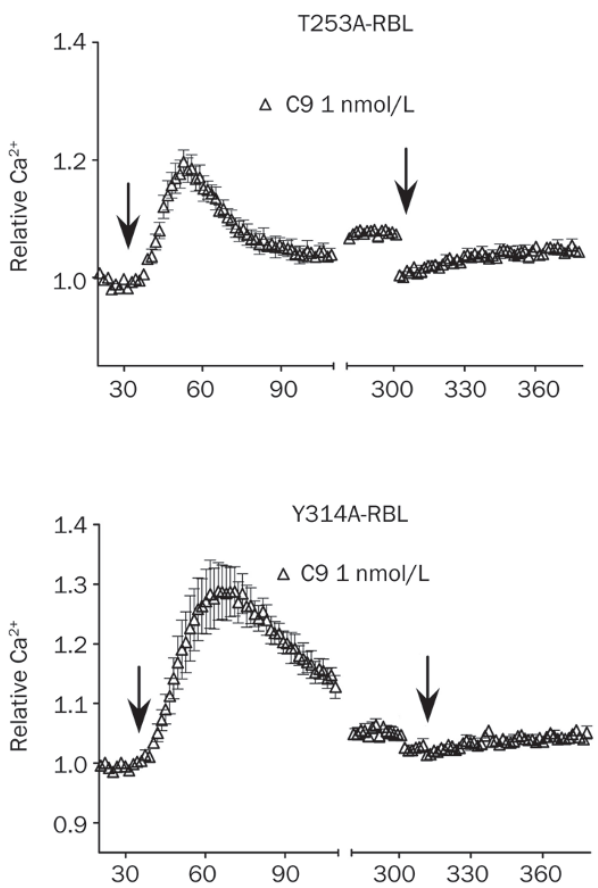

S347A-RBI

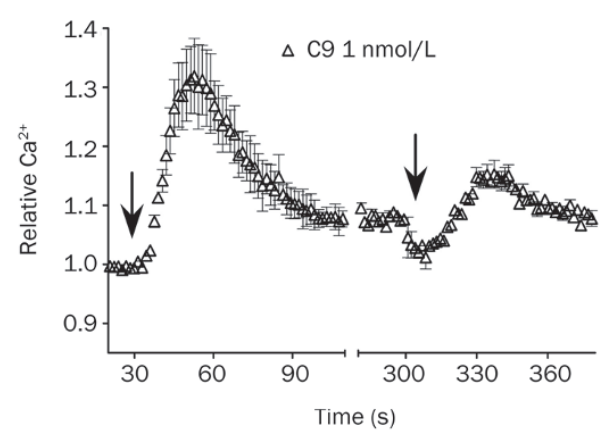

Figure 6. Calcium mobilization in cells expressing WT and mutant CMKLR1. (A) HEK 293 cells were transiently transfected with constructs encoding WT human CMKLR1 or different mutants (T253A, Y314A, or S347A) that affected receptor internalization (Figure 5). The cells were loaded with FLIPR Calcium 5 reagent $36 \mathrm{~h}$ after transfection. Agonist (C9; $1 \mathrm{nmol} / \mathrm{L})$ was added at the time indicated by the arrows. The traces represent the real-time changes in the intracellular calcium concentration, which is shown as the ratio of measurement at $525 \mathrm{nmol} / \mathrm{L}$ to the baseline and is expressed as the mean \pm SEM of two separate experiments, each experiment was carried out in triplicate. (B) Same as in A but using stable RBL transfectants for each mutants. A second stimulation of $\mathrm{C} 9$ was applied $260 \mathrm{~s}$ after the first stimulation. Note that the $\mathrm{Ca}^{2+}$ traces returned to baseline sooner than that in HEK 293 after stimulation. Data are expressed similarly to those in A above.

and 347 (predicted PKC site) also abrogated receptor internalization. In comparison, alanine substitution of other potential sites for GRK (Ser337) and PKC (Ser344) did not alter receptor internalization. Interestingly, alanine substitution of Thr253, 
which does not match any consensus sequence for the above protein kinases, also blocked receptor internalization (Figure 5). These results are summarized in Table 1.

\section{Effects of receptor internalization on CMKLR1-mediated func- tions}

Having generated a number of mutant receptor constructs, we sought to determine whether CMKLR1 internalization (or lack thereof) had any impact on receptor-mediated functions. Calcium mobilization is an early response mediated by a subclass of GPCRs, including CMKLR1. The four mutant constructs with altered receptor internalization were expressed in HEK 293 cells, and agonist-induced changes in intracellular $\mathrm{Ca}^{2+}$ concentration were measured. As shown in Figure 6A, none of the internalization-deficient mutations abrogated the C9-induced $\mathrm{Ca}^{2+}$ mobilization. The result suggests that receptor internalization is not a prerequisite for agonist-induced $\mathrm{Ca}^{2+}$ mobilization. A close examination identified increased $\mathrm{Ca}^{2+}$ influx with some of the mutants such as S347A, which may result from a loss of receptor desensitization. This possibility was tested in stable RBL transfectants expressing the individual mutants. As shown in Figure 6B, a second $\mathrm{Ca}^{2+}$ flux $260 \mathrm{~s}$ after the first stimulation was observed, suggesting a partial loss of desensitization. However, this was not seen with the other mutants tested. In parallel experiments, C15 was examined but found unable to stimulate $\mathrm{Ca}^{2+}$ flux (data not shown).

We next examined whether receptor internalization is involved in the agonist-induced ERK phosphorylation. Published reports have indicated that $\beta$-adrenergic receptor internalization is required for ERK phosphorylation in a tryosineprotein kinase-dependent manner ${ }^{[36]}$. However, subsequent studies have shown that this requirement does not apply to several other GPCRs ${ }^{[37,38]}$. In transfected cells expressing WT CMKLR1, C9 induced the rapid phosphorylation of ERK 1/2, which became apparent at $2 \mathrm{~min}$ and peaked at $5 \mathrm{~min}$. Phosphorylation of ERK $1 / 2$ subsided after 5 min (Figure 7). ERK $1 / 2$ phosphorylation did not seem to require the internalization of CMKLR1, as the level of phosphorylation remained similar in the cells expressing the internalization-deficient mutants (T253A, Y314A, S343A, and S347A). Close examination revealed changes in the kinetics of ERK $1 / 2$ phosphorylation, with peak phosphorylation occurring at 2 min instead of $5 \mathrm{~min}$. To determine whether there is any link between receptor internalization and the left-shift in the time course, we examined the two mutants that did not abrogate receptor internalization (S350A and T352A). As shown in Figure 7, the time course of ERK phosphorylation in cells expressing these
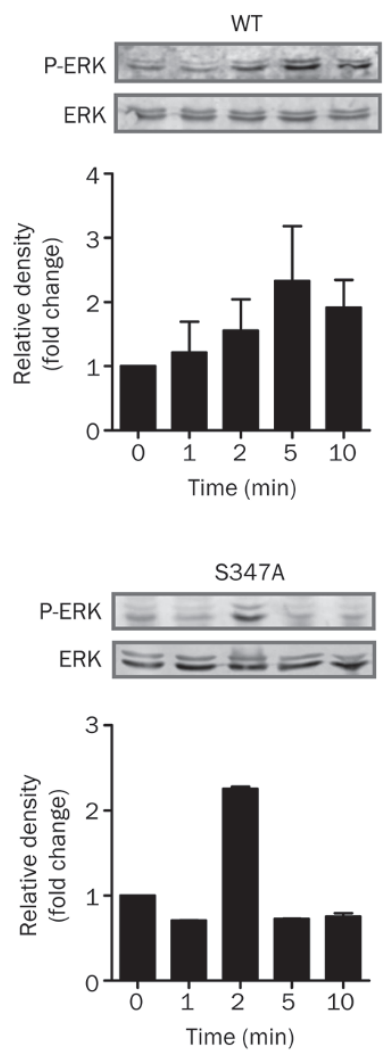
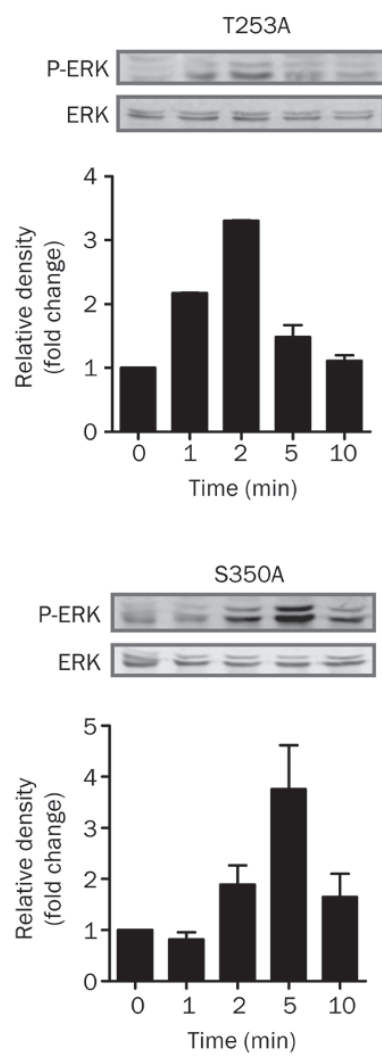
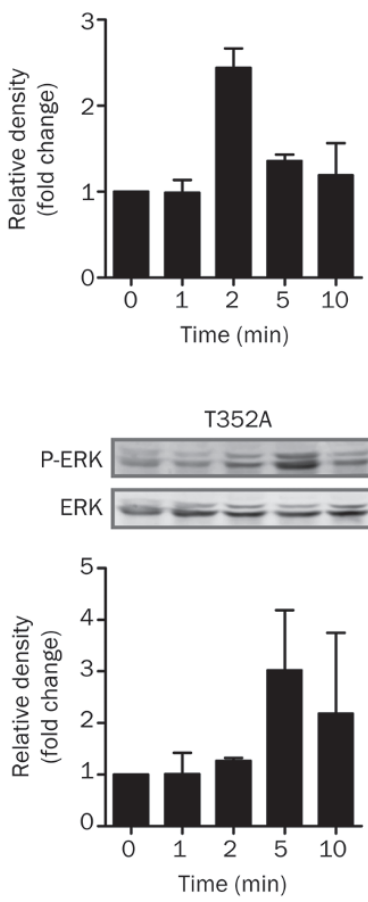
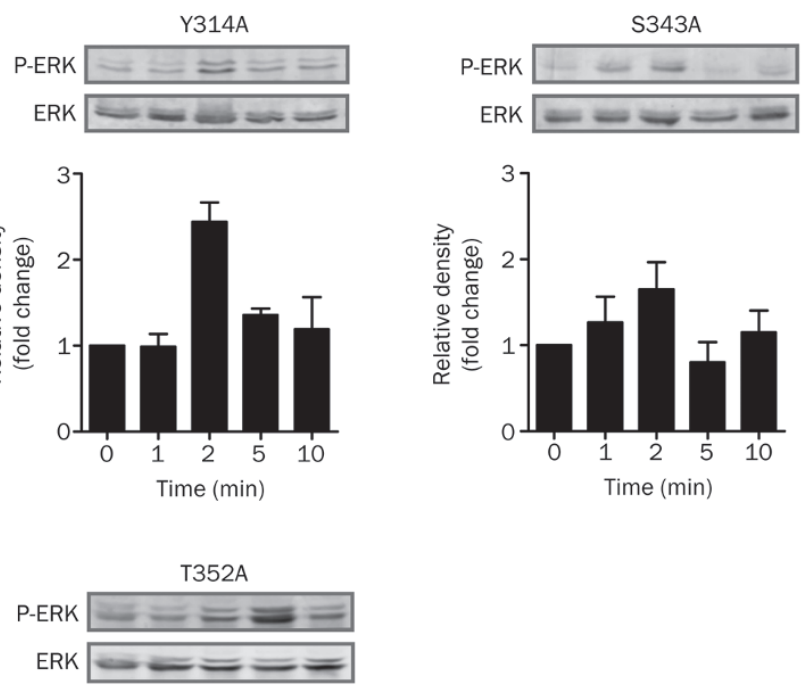

Figure 7. Effect of CMKLR1 point mutations on the agonist-induced phosphorylation of ERK 1/2. Transiently transfected HeLa cells expressing either WT or mutant CMKLR1 receptors were harvested $36 \mathrm{~h}$ after transfection. The cells were stimulated with $10 \mathrm{nmol} / \mathrm{L} \mathrm{C9}$ at $37^{\circ} \mathrm{C}$ for the indicated lengths of time. The samples were separated by SDS-PAGE and blotted onto PVDF membranes, and p-ERK and total ERK were detected using an antiphospho-ERK antibody and an anti-ERK antibody, respectively. Representative blots from at least 3 experiments are shown. The relative density of the ERK bands was quantified and is shown in the bar graphs (the mean \pm SEM) below the individual blots. 
internalization-capable mutants was similar to that in cells with the WT CMKLR1. However, the sample size was insufficient to conclude that these kinetic changes were significant.

\section{Discussion}

In this study, we examined the internalization of the chemerin receptor CMKLR1, a GPCR. Our results show that CMKLR1 internalization is triggered by full-length chemerin and by the C9 peptide which retains the activity of chemerin. The C9 peptide induced the dose-dependent internalization of CMKLR1 that was readily detectable at $1 \mathrm{nmol} / \mathrm{L}$. At a higher concentration of C9 $(1 \mu \mathrm{mol} / \mathrm{L})$, approximately $90 \%$ of the receptors were cleared from the cell surface, suggesting that highly efficient endocytosis occurred following C9 stimulation. Our results show that intact CMKLR1 without the EGFP fusion was internalized after C9 stimulation, demonstrating that fusion of the receptor with EGFP did not alter CMKLR1 internalization. Moreover, the C9-induced CMKLR1 internalization was similar in the three cell lines tested, confirming that it is not cell type-specific. In contrast to C9, the C15 peptide failed to stimulate the internalization of CMKLR1. C15 was generated by cysteine proteases, following the activation of prochemerin by serine proteases ${ }^{[5]}$. Previous studies have shown that $\mathrm{C} 15$ lacks the ability to induce calcium mobilization and chemotaxis through CMKLR $1^{[15,16]}$, but possesses its anti-inflammatory functions ${ }^{[17]}$. Of note, we found that the addition of the $\mathrm{C} 15$ peptide prior to the $\mathrm{C} 9$ peptide or chemerin abrogated CMKLR1 internalization. This effect was not observed with sC15, a 15-residue peptide with scrambled sequence, suggesting that $\mathrm{C} 15$ might be an antagonist that blocks the action of $\mathrm{C} 9$ and chemerin. One published report indicated that the $\mathrm{C} 15$ peptide may potentiate the phagocytosis of bacterial particles and apoptotic cells by macrophages ${ }^{[17]}$. Further investigation should determine whether $\mathrm{C} 15$ is a partial agonist for CMKLR1, but our data clearly show that it is not effective in stimulating CMKLR1 internalization even at micromolar concentrations.

To date, three chemerin receptors have been identified. Although all of these receptors (CMKLR1, GPR1, and CCRL2) bind chemerin with high affinity ${ }^{[5,25,39]}$, only CMKLR1 has been shown to be a fully functional receptor for chemerin. Exactly how these receptors behave differently remains unclear. In this study, we examined the structural determinants for CMKLR1 internalization. The C-terminal domain of CMKLR1 contains the necessary structures for receptor internalization, including the NPXXY motif and the consensus sites for GRK and PKC phosphorylation. The present study was not intended as a thorough investigation of CMKLR1 phosphorylation; however, selective mutations of putative phosphorylation sites in CMKLR1 led to the identification of sites important for receptor internalization. Alanine substitutions at selected sites effectively abrogated CMKLR1 internalization, suggesting that these potential phosphorylation sites work together during receptor internalization. In a study of formyl peptide receptor 1 (FPR1) internalization, it was discovered that sequential phosphorylation of its C-terminal domain was important for FPR1 internalization, and deletion of one site could affect the phosphorylation of another site ${ }^{[23]}$. Further examination of the potential phosphorylation sites in CMKLR1 is required to fully delineate the mechanism of its internalization.

Agonist-induced internalization is a major mechanism for the clearance of cell surface receptors. Several chemokine receptors, including DARC (Duffy antigen/receptor for chemokines), D6 and CCX-CKR, lack the signature sequence for G protein coupling (DRYLAIV). As a result, the primary function of these receptors is clearance of chemokines from their surrounding environment ${ }^{[19]}$. As for chemerin, one of its receptors, GPR1, is a high affinity receptor but lacks the ability to efficiently transduce signals ${ }^{[39]}$. Likewise, CCRL2 is devoid of signaling capability seen in a typical GPCR. However, it also fails to provide ligand-scavenging function $\mathrm{s}^{[40]}$. In contrast, the chemerin receptor CMKLR1 is capable of internalization, as well as transmembrane signaling. For these GPCRs, internalization is more than a mechanism for ligand removal; it is closely associated with receptor signaling. Previous reports have indicated a requirement of GPCR (eg, $\beta$-adrenergic receptor) internalization for ERK phosphorylation ${ }^{[36]}$. In the present study, CMKLR1 internalization did not appear to be required for ERK phosphorylation; instead, the absence of CMKLR1 internalization altered the kinetics of ERK phosphorylation by reducing the time needed to reach peak phosphorylation. These results suggest that the presence of CMKLR1 on the cell surface is important for proper signaling, and its internalization provides a negative regulatory mechanism for agonistinduced signaling. The observation that the Y314A mutation, which affects receptor internalization, results in an ERK phosphorylation pattern similar to those of the mutations targeting the protein kinase phosphorylation sites strongly suggests that the removal of CMKLR1 from the cell surface might be responsible for the late appearance ( $5 \mathrm{~min}$ instead of $2 \mathrm{~min}$ ) of the ERK phosphorylation peak. Additional experiments are required for a more detailed analysis of how CMKLR1 phosphorylation affects its signaling.

In conclusion, the present study shows that the two chemerin-derived peptides, C9 and C15, function differently in triggering CMKLR1 internalization and demonstrates that multiple phosphorylation events are required for efficient internalization of the receptor, which occurs through a clathrin-independent pathway. These findings provide a foundation for more detailed investigation of CMKLR1 internalization and signaling in the future.

\section{Acknowledgements}

This work was supported, in part, by the National Natural Science Foundation of China (Grant 31270941), the National Basic Research Program of China (973 Program Grant 2012CB518000), the Specialized Research Fund for the Doctoral Program of Higher Education of China (Grant 20120073110069), and the US National Institutes of Health (Grant R01 AI 033503). 


\section{Author contribution}

Richard D YE and Hui-qiong HE designed the research; Junxian ZHOU, Dan LIAO, and Shuo ZHANG performed the research; Jun-xian ZHOU, Hui-qiong HE, and Dan LIAO analyzed the data; Ni CHENG contributed critical reagents; and Richard D YE, Jun-xian ZHOU, and Hui-qiong HE wrote the paper.

\section{References}

1 Gantz I, Konda Y, Yang YK, Miller DE, Dierick HA, Yamada T. Molecular cloning of a novel receptor (CMKLR1) with homology to the chemotactic factor receptors. Cytogenet Cell Genet 1996; 74: 286-90.

2 Samson M, Edinger AL, Stordeur P, Rucker J, Verhasselt V, Sharron M, et al. ChemR23, a putative chemoattractant receptor, is expressed in monocyte-derived dendritic cells and macrophages and is a coreceptor for SIV and some primary HIV-1 strains. Eur J Immunol 1998; 28: 1689-700.

3 Wittamer V, Franssen JD, Vulcano M, Mirjolet JF, Le Poul E, Migeotte I, et al. Specific recruitment of antigen-presenting cells by chemerin, a novel processed ligand from human inflammatory fluids. J Exp Med 2003; 198: 977-85.

4 Meder W, Wendland M, Busmann A, Kutzleb C, Spodsberg N, John H, et al. Characterization of human circulating TIG2 as a ligand for the orphan receptor ChemR23. FEBS Lett 2003; 555: 495-9.

5 Bondue B, Wittamer V, Parmentier M. Chemerin and its receptors in leukocyte trafficking, inflammation and metabolism. Cytokine Growth Factor Rev 2011; 22: 331-8.

6 Zabel BA, Allen SJ, Kulig P, Allen JA, Cichy J, Handel TM, et al. Chemerin activation by serine proteases of the coagulation, fibrinolytic, and inflammatory cascades. J Biol Chem 2005; 280: 34661-6.

7 Guillabert A, Wittamer V, Bondue B, Godot V, Imbault V, Parmentier $\mathrm{M}$, et al. Role of neutrophil proteinase 3 and mast cell chymase in chemerin proteolytic regulation. J Leukoc Biol 2008; 84: 1530-8.

8 Wittamer V, Bondue B, Guillabert A, Vassart G, Parmentier M, Communi D. Neutrophil-mediated maturation of chemerin: a link between innate and adaptive immunity. J Immunol 2005; 175: 487 93.

9 Vermi W, Riboldi E, Wittamer V, Gentili F, Luini W, Marrelli S, et al. Role of ChemR23 in directing the migration of myeloid and plasmacytoid dendritic cells to lymphoid organs and inflamed skin. J Exp Med 2005; 201: 509-15.

10 Parolini S, Santoro A, Marcenaro E, Luini W, Massardi L, Facchetti F, et al. The role of chemerin in the colocalization of NK and dendritic cell subsets into inflamed tissues. Blood 2007; 109: 3625-32.

11 Arita M, Ohira T, Sun YP, Elangovan S, Chiang N, Serhan CN. Resolvin E1 selectively interacts with leukotriene B4 receptor BLT1 and ChemR23 to regulate inflammation. J Immunol 2007; 178: 3912-7.

12 Goralski KB, McCarthy TC, Hanniman EA, Zabel BA, Butcher EC, Parlee $\mathrm{SD}$, et al. Chemerin, a novel adipokine that regulates adipogenesis and adipocyte metabolism. J Biol Chem 2007; 282: 28175-88.

13 Bozaoglu K, Bolton K, McMillan J, Zimmet P, Jowett J, Collier G, et al. Chemerin is a novel adipokine associated with obesity and metabolic syndrome. Endocrinology 2007; 148: 4687-94.

14 Wittamer V, Gregoire F, Robberecht P, Vassart G, Communi D, Parmentier M. The C-terminal nonapeptide of mature chemerin activates the chemerin receptor with low nanomolar potency. J Biol Chem 2004; 279: 9956-62.

15 Luangsay S, Wittamer V, Bondue B, De Henau O, Rouger L, Brait M, et al. Mouse ChemR23 is expressed in dendritic cell subsets and macrophages, and mediates an anti-inflammatory activity of chemerin in a lung disease model. J Immunol 2009; 183: 6489-99.

16 Shimamura K, Matsuda M, Miyamoto Y, Yoshimoto R, Seo T, Tokita S. Identification of a stable chemerin analog with potent activity toward ChemR23. Peptides 2009; 30: 1529-38.

17 Cash JL, Hart R, Russ A, Dixon JP, Colledge WH, Doran J, et al. Synthetic chemerin-derived peptides suppress inflammation through ChemR23. J Exp Med 2008; 205: 767-75.

18 Cash JL, Christian AR, Greaves DR. Chemerin peptides promote phagocytosis in a ChemR23- and Syk-dependent manner. J Immunol 2010; 184: 5315-24

19 Koenig JA, Edwardson JM. Endocytosis and recycling of G proteincoupled receptors. Trends Pharmacol Sci 1997; 18: 276-87.

20 von Zastrow $M$. Mechanisms regulating membrane trafficking of $G$ protein-coupled receptors in the endocytic pathway. Life Sci 2003; 74: $217-24$

21 Ostrom RS, Insel PA. The evolving role of lipid rafts and caveolae in $G$ protein-coupled receptor signaling: implications for molecular pharmacology. Br J Pharmacol 2004; 143: 235-45.

22 Tardif M, Mery L, Brouchon L, Boulay F. Agonist-dependent phosphorylation of $\mathrm{N}$-formylpeptide and activation peptide from the fifth component of $\mathrm{C}$ (C5a) chemoattractant receptors in differentiated HL60 cells. J Immunol 1993; 150: 3534-45.

23 Prossnitz ER, Kim CM, Benovic JL, Ye RD. Phosphorylation of the $\mathrm{N}$-formyl peptide receptor carboxyl terminus by the $\mathrm{G}$ protein-coupled receptor kinase, GRK2. J Biol Chem 1995; 270: 1130-7.

24 Ferguson SS, Downey WE 3rd, Colapietro AM, Barak LS, Ménard L, Caron MG. Role of beta-arrestin in mediating agonist-promoted G protein-coupled receptor internalization. Science 1996; 271: 363-6.

25 Zabel BA, Nakae S, Zuniga L, Kim JY, Ohyama T, Alt C, et al. Mast cellexpressed orphan receptor CCRL2 binds chemerin and is required for optimal induction of IgE-mediated passive cutaneous anaphylaxis. J Exp Med 2008; 205: 2207-20.

26 Monnier J, Lewen S, O'Hara E, Huang K, Tu H, Butcher EC, et al. Expression, regulation, and function of atypical chemerin receptor CCRL2 on endothelial cells. J Immunol 2012; 189: 956-67.

27 Phonphok Y, Rosenthal KS. Stabilization of clathrin coated vesicles by amantadine, tromantadine and other hydrophobic amines. FEBS Lett 1991; 281: 188-90.

28 Tse SM, Furuya W, Gold E, Schreiber AD, Sandvig K, Inman RD, et al. Differential role of actin, clathrin, and dynamin in Fc gamma receptormediated endocytosis and phagocytosis. J Biol Chem 2003; 278: 3331-8.

29 McGookey DJ, Fagerberg K, Anderson RG. Filipin-cholesterol complexes form in uncoated vesicle membrane derived from coated vesicles during receptor-mediated endocytosis of low density lipoprotein. J Cell Biol 1983; 96: 1273-8.

30 Schnitzer JE, Oh P, Pinney E, Allard J. Filipin-sensitive caveolaemediated transport in endothelium: reduced transcytosis, scavenger endocytosis, and capillary permeability of select macromolecules. J Cell Biol 1994; 127: 1217-32.

31 Benovic JL, Kuhn H, Weyand I, Codina J, Caron MG, Lefkowitz RJ. Functional desensitization of the isolated beta-adrenergic receptor by the beta-adrenergic receptor kinase: potential role of an analog of the retinal protein arrestin (48-kDa protein). Proc Natl Acad Sci U S A 1987; 84: 8879-82.

32 Lohse MJ, Benovic JL, Codina J, Caron MG, Lefkowitz RJ. betaArrestin: a protein that regulates beta-adrenergic receptor function. Science 1990; 248: 1547-50.

33 Rapacciuolo A, Suvarna S, Barki-Harrington L, Luttrell LM, Cong M, 
Lefkowitz RJ, et al. Protein kinase A and G protein-coupled receptor kinase phosphorylation mediates beta- 1 adrenergic receptor endocytosis through different pathways. J Biol Chem 2003; 278 : 35403-11.

34 Xue Y, Li A, Wang L, Feng H, Yao X. PPSP: prediction of PKspecific phosphorylation site with Bayesian decision theory. BMC Bioinformatics 2006; 7: 163.

35 Barak LS, Tiberi M, Freedman NJ, Kwatra MM, Lefkowitz RJ, Caron $M G$. A highly conserved tyrosine residue in $G$ protein-coupled receptors is required for agonist-mediated beta 2-adrenergic receptor sequestration. J Biol Chem 1994; 269: 2790-5.

36 Daaka Y, Luttrell LM, Ahn S, Della Rocca GJ, Ferguson SS, Caron MG, et al. Essential role for $\mathrm{G}$ protein-coupled receptor endocytosis in the activation of mitogen-activated protein kinase. J Biol Chem 1998; 273: $685-8$.
37 Schramm NL, Limbird LE. Stimulation of mitogen-activated protein kinase by $\mathrm{G}$ protein-coupled alpha(2)-adrenergic receptors does not require agonist-elicited endocytosis. J Biol Chem 1999; 274: 2493540.

38 Kim SJ, Kim MY, Lee EJ, Ahn YS, Baik JH. Distinct regulation of internalization and mitogen-activated protein kinase activation by two isoforms of the dopamine D2 receptor. Mol Endocrinol 2004; 18: 640-52.

39 Barnea G, Strapps W, Herrada G, Berman Y, Ong J, Kloss B, et al. The genetic design of signaling cascades to record receptor activation. Proc Natl Acad Sci U S A 2008; 105: 64-9.

40 Del Prete A, Bonecchi R, Vecchi A, Mantovani A, Sozzani S. CCRL2, a fringe member of the atypical chemoattractant receptor family. Eur J Immunol 2013; 43: 1418-22. 